\title{
Imagerie micronique à l'aide de lentilles à zones de Fresnel
}

P. Troussel, M. Pichet, J.M. Dalmasso, M. Idir*, T. Jalinaud, M. Ollivier, J.P. Le Breton et R. Sauneuf

Commissariat à l'Énergie Atomique, CEA/CEL-V, 94195 Villeneuve-Saint-Georges cedex, France

* Laboratoire de Spectroscopie Atomique et Ionique, Université Paris-Sud, 91405 Orsay

cedex, France

Résumé : Nous présentons une étude d“imagerie X par lentilles à zones de Fresnel dans le domaine 1-5 keV. Les résolutions spatiales obtenues par des images radiographiques de grilles sont évaluées à quelques microns à l'aide de trois sources X diffétentes.

\section{INTRODUCTION}

Les lentilles diffractives à zones de Fresnel (LZF) utilisables dans le domaine des rayons X sont composées d'anneaux circulaires concentriques successifs alternativement transparents et semi-opaques . Le premier anneau est en réalité un disque de rayon $R_{1}$ et le rayon extérieur de l'anneau $n^{\circ} i$ est $R_{i}=R_{1} V_{i}$.

Utilisées en transmission elles se comportent comme les lentilles classiques en verre utilisées dans le domaine visible mais présentent une aberration chromatique importante. Leur résolution spatiale élevée ( voisine de la largeur du dernier anneau) explique leur utilisation depuis plus d'une dizaine d'années en microscopie biologique avec un rayonnement monochromatique d'énergie voisine de $275 \mathrm{eV}$ (fenêtre de l'eau). Elles peuvent cependant avoir des applications analogues dans l'étude des plasmas laser avec des rayons $\mathrm{X}$ d'énergie plus élevées ( 1 à $10 \mathrm{keV})$.

Il existe deux types de LZF:

- les lentilles dites d'amplitude (LZFA) où les anneaux semi-opaques sont, en réalité, totalement opaques; leur efficacité maximale est d'environ $10 \%$ (1)

- les lentilles dites de phase (LZFP) où les anneaux semi-opaques occasionnent un déphasage de $\pi$ au rayonnement qui les traverse; leur efficacité peut alors atteindre $40 \%$ (2).

Nous présentons une étude expérimentale réalisée avec une LZFP en or fabriquée par lithographie (2). Elle comporte 68 anneaux de rayons $R_{1}=6,63 \mu \mathrm{m}$ et $R n=55 \mu \mathrm{m}$; ce dernier anneau a une largeur $\Delta R=0,4 \mu \mathrm{m}$; les anneaux semi-opaques ont un épaisseur de $0,5 \mu \mathrm{m}$.

\section{RESULTATS}

Trois types de sources $\mathrm{X}$ ont été utilisées lors de cette étude:

\section{1. Rayonnement synchrotron}

Cette source de par sa forte brillance et sa haute monochromaticité est un outil de choix pour caractériser ces optiques focalisantes dans des conditions quasi-idéales. Les performances de notre LZFP ont été évaluées à l'aide de la ligne de lumière SB3 (LURE/Orsay) équipée d'un monochromateur à double cristal et d'un goniomètre situé à 14 mètres du point source. Le détecteur utilisé pour mesurer les efficacités de la lentille, est un détecteur à flux gazeux avec un sténopé de $15 \mu \mathrm{m}$ placé à la distance focale de la zone correspondant à l'énergie de photons. L'efficacité, maximum, à l'ordre 1 a été obtenue à $1900 \mathrm{eV}$, énergie où l'effet de phase est le plus grand et évaluée à $23 \%$, conformèment à la théorie, le contraste entre l'ordre 1 et l'ordre 0 est alors de 55 . L'image radiographique à $1430 \mathrm{eV}$ d'une grille de $25,4 \mu \mathrm{m}$ au grandissement 44 placée à proximité du foyer (focale $=5,18 \mathrm{~cm}$ ) est représentèe sur la 
figure 1, ainsi qu'une coupe transversale est donnée. La résolution est évaluée à moins de $1 \mu \mathrm{m}$ dans le plan objet par la méthode du couteau sur les barreaux de la grille: cela consiste à considérer que la résolution spatiale ultime est la hauteur de ces pics de dérivée.
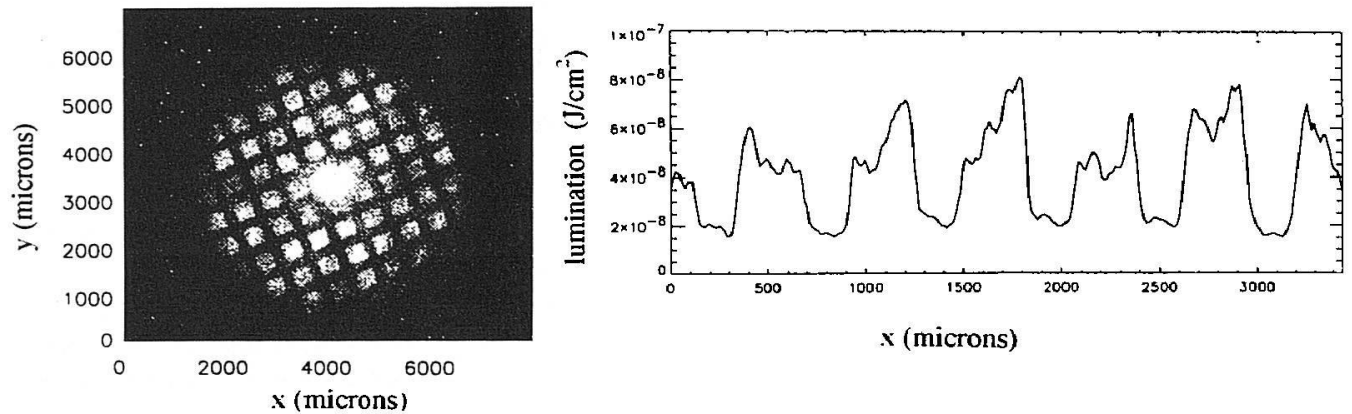

Figure 1 :Radiographie d'une grille de $25,4 \mu \mathrm{m}$ avec un faisceau synchrotron à $1430 \mathrm{eV}$ et parallèle.

\section{2. Source continue de type Henke}

Cette source à bombardement électronique a permis d'évaluer, par cette même méthode, à $8 \mu \mathrm{m}$ la résolution de la zone pour un champ voisin de $1 \mathrm{~mm}$. Cette résolution est moins bonne car le spectre (continuum et raies) est relativement étendu et la source spatialement non ponctuelle : il subsiste des aberrations notamment chromatiques.

\section{3. Source plasma laser}

Nous avons utilisé l'installation Heliotrope-Octal pour former un plasma de gadolinium de $200 \mu \mathrm{m}$ de diamètre, dont le rayonnement a un spectre plus étroit que celui de la source Henke ( bande de $65 \mathrm{eV}$ à mi-hauteur centrée sur $1430 \mathrm{eV}$ ). La résolution spatiale est de l'ordre de $5 \mu \mathrm{m}$. En interposant un miroir multicouche sélectif, ( 200 paires W/Si de période $26 \mathrm{~A}$ de période donnant $\mathrm{E} / \Delta \mathrm{E}=70$ ), on réduit la bande spectrale utile et on améliore notablement la résolution que l'on peut évaluer à $4 \mu \mathrm{m}$ (Fig. 2).
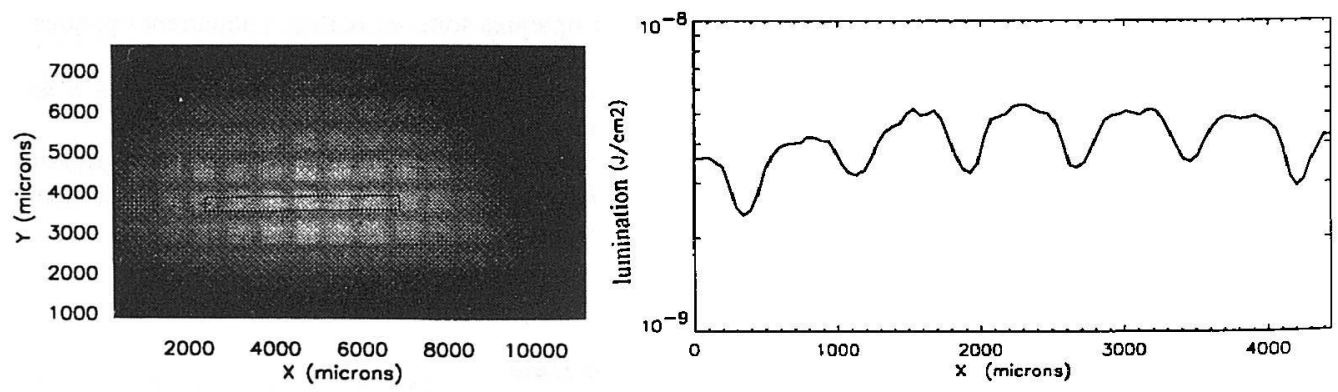

Figure 2 : Radiographie d'une grille de $25,4 \mu \mathrm{m}$ avec la source $\mathrm{X}$ divergente d'un plasma de gadolinium

\section{CONCLUSION}

Les premiers tests sur ces sources sont encourageants, les améliorations de cette résolution, loin de la résolution spatiale minimale fixée par la taille du dernier anneau $(0.5 \mu \mathrm{m})$, pourront être la réalisation de lentilles dites hors axe, la suppression radicale de l'aberration chromatique par un miroir encore plus sélectif ou par un réseau gravé multicouche, et aussi la réalisation de substrats des miroirs avec une excellente planéité. Ces améliorations se feront cependant au détriment de la sensibilité.

\section{Références}

1. G. Schmahl, X-ray microscopy : Nucl. Inst. Meth. 208 (1983) 361-365

2. Heidenhain Belgien Bellekouter, 30, B-1790 Affligem. 